\title{
A REVIEW OF DIVERSITY IN THE EVOLUTION AND DEVELOPMENT OF CARTILAGE: THE SEARCH FOR THE ORIGIN OF THE CHONDROCYTE
}

\author{
A.G. Cole* \\ IRGS - BioGem s.c.a.r.1., I-83031 Ariano Irpino, AV, Italy
}

\begin{abstract}
Mammalian cartilage is a complex and developmentally important tissue type. Outside the mammalian lineage, cartilage may persist as an adult tissue, which shows a much wider diversity of histological structure. Tissues similar to vertebrate cartilage are also found within multiple invertebrate lineages, including mollusks, arthropods, and polychaetes, however the relationship of these tissues to vertebrate cartilage is unknown. Detailed molecular analysis of these invertebrate tissues is necessary to assess the degree of homology, if any, of cartilage throughout the metazoans. The purpose of the following review is to introduce readers to this diversity of cartilage and to synthesize the known genetic interactions that give rise to vertebrate cartilage into the format of a gene regulatory network (GRN). This chondrogenesis GRN highlights a large number of transcription factors known to be expressed during chondrogenesis, whose role in this process has yet to be elucidated. Verification and expansion of this initial GRN will assist in the identification of the core set of the genetic interactions necessary for the specification of the vertebrate chondrocyte. This is the necessary first step in allowing detailed comparison of the molecular signature of vertebrate chondrocytes with that of invertebrates with the ultimate goal of understanding the evolutionary origin of this important skeletal cell type.
\end{abstract}

Keywords: Gene regulatory network, molecular fingerprint, invertebrate cartilage, cell type evolution.

*Address for correspondence:

A.G. Cole

IRGS - BioGem s.c.a.r.l.

COGED

Via Camporeale Area P.I.P.

I-83031 Ariano Irpino, AV, Italy

E-mail: agcole@gmail.com

\section{Introduction}

Historically cartilage has been defined as a vertebrate tissue that forms part of the skeletal system that is grossly different from mineralized bone both in function and histological composition. Cartilage can be defined generally as an internal cellular support tissue high in fibrous protein and mucopolysaccharide content (see Cole and Hall, 2004a, for discussion). In mammals 3 classic types of cartilage have been histologically identified based upon the relative contribution and distribution of fibers within the extracellular matrix: hyaline cartilage is the typical cartilage that is generally referred to when one thinks of cartilage. Elastic cartilage contains additional elastin fibers, and fibrocartilage contains regions of organized fibrous tissue within the extracellular matrix. Developmentally, cartilage can be followed histologically through three general phases (Hall and Miyake, 2000). Prior to cell differentiation, within the mesenchyme a cellular condensation forms - known as the cartilage anlagen (Cameron et al., 2010), protocartilage (Cole and Hall, 2009), or cartilage condensation (Hall and Miyake, 2000). These cells then begin to secrete the cartilagespecific matrix. As development progresses the chondrocytes continue to proliferate and generate extracellular matrix, which then calcifies as the cells enter the hypertrophic phase of differentiation. This calcified cartilage matrix is then replaced by bone - in a process known as endochondral bone formation. The purpose of this review is to introduce readers to the diversity that exists outside this general description of cartilage, and to synthesize what is known about the molecular nature of chondrocyte specification as a means to address the origin of this important cell type.

\section{Diversity of Cartilage and Cartilage-like Tissues}

Although hyaline cartilage is the most well-studied cartilaginous tissue both due to its developmental importance in laying down the scaffold for most of the major bones of the body, and due to the fact that cartilage is retained at the joint interfaces and is the primary tissue affected in arthritis - there also exists a large variety of histologically diverse cartilage types that persist as adult tissues, particularly found within fish lineages. Benjamin (1990) attempts to catalog this diversity, illustrating cartilages that vary in the relative amount of matrix with respect to cell density, and the presence of variation of components within the extracellular matrix. This system of cartilage classification has recently been re-visited (Witten et al., 2010) wherein the authors stress that on 


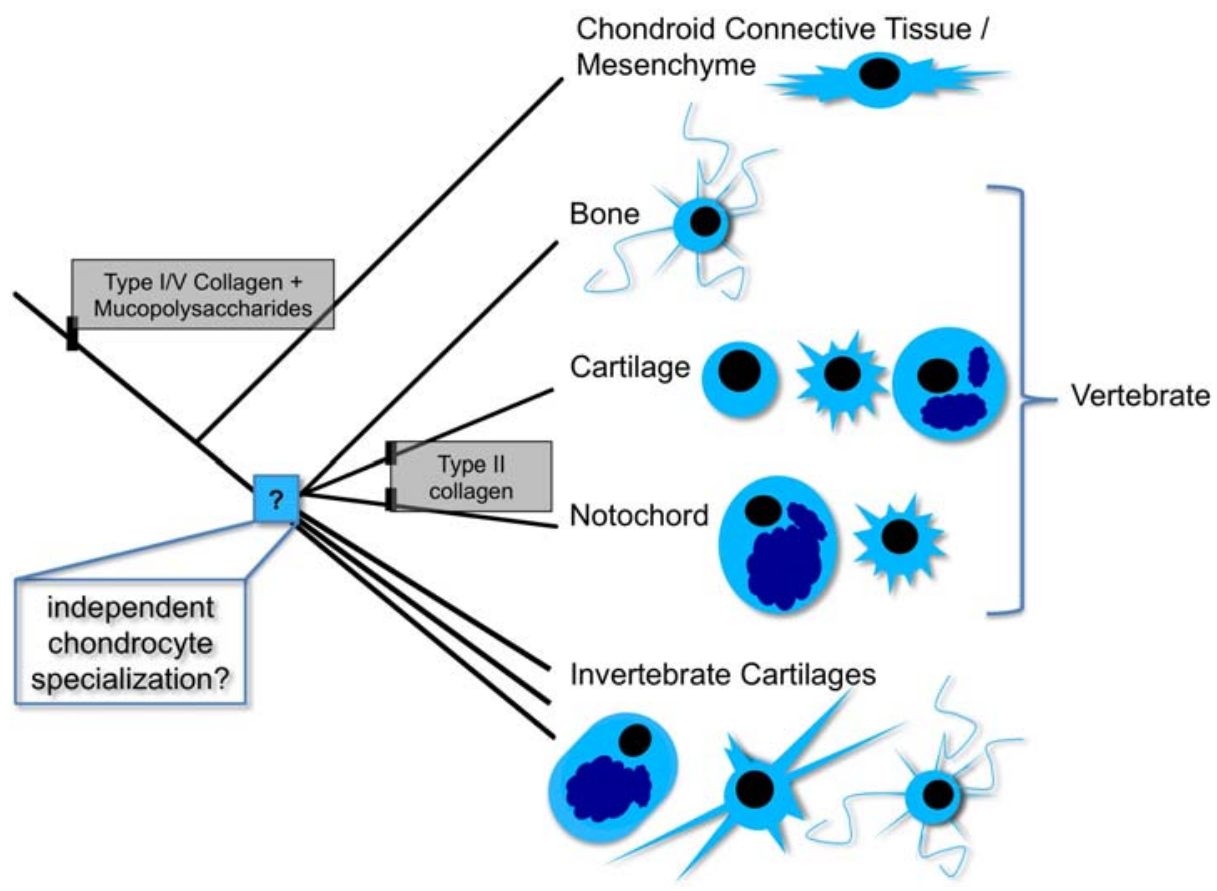

Fig. 1. Schematic depicting the problem the evolutionary relations between various connective tissues. Deposition of fibrous collagens and mucopolysaccharides in a structured connective tissue is an ancestral feature, whereas type II collagen is known to be specific to the vertebrate radiation and is present in both vertebrate cartilage and notochord. At present it is unclear whether the chondrocyte has evolved multiple times, or if there was a single origin and subsequent diversification of this cell type.

the basis of histology, cartilage is best considered as a spectrum of connective tissue types.

Interestingly, cartilage-like tissues have also been described in a variety of invertebrate lineages. Person and Philpot (1969) make the following statement regarding cartilage: "there is a body of information which suggest that just as nature knew how to form muscle, nerve, gland, and other tissues in the invertebrates, she also knew how to make cartilage, perhaps even before vertebrates appeared on the evolutionary scene" (pg. 2). The most compelling evidence to which the authors refer, is the histological organization of these invertebrate tissues. In fact within the invertebrates that have been investigated, a spectrum of histological tissues that parallels the diversity found within modern fishes is found (Cole and Hall, 2004b). Cartilages found within Cephalopod mollusks are remarkably similar to vertebrate hyaline cartilage at a histological level (Cole and Hall, 2004a). On close inspection, one can find one significant difference between vertebrate hyaline cartilage and that found within the cephalopod - the extra-cellular matrix of the cephalopod, in addition to the chondrocytes, is riddled with small canals - through which pass thin extensions of chondrocyte processes which retain connections between one another (Bairati et al., 1998; Leone et al., 2004; Cole and Hall, 2009). These cellular processes are a feature of vertebrate osteocytes rather than chondrocytes, although cell processes have been recently described within a subset of human chondrocytes using confocal microscopy to image live chondrocytes (Murray et al., 2010), and chondrocytes require a high density three dimensional culture environment to retain their spherical morphology (Abbott et al., 1966); when cultured in two dimensions, chondrocytes revert to a fibroblast-like morphology and stop producing cartilage extracellular matrix (Chacko et al., 1969). The physiological significance of these phenomena, if any, is currently unknown.

Another interesting feature of some cartilage-like tissues found within various invertebrate lineages is the presence of vacuolated cells (Cole and Hall, 2004b). For example, the polychaete family Sabellidae is defined by the presence of a cellular cartilage-like skeleton within its feeding tentacles (Kupriyanova and Rouse, 2008). This tissue is highly cellular, and the cells appear contain large vesicles (Cole and Hall, 2004b). Although hypertrophic chondrocytes are also characterized by an increase in cell volume that is accompanied by the accumulation of material within large vesicles, this is a histological feature that parallels the notochord in structure more so than the typical hyaline chondrocyte.

The vertebrate notochord produces many of the same extracellular matrix molecules as cartilage (for example Type II collagen), however at a much lower quantity, and the cells are large and vacuolated, surrounded by a mesenchymal-derived perinotochordal sheath. The affinity of the notochord to cartilage remains debatable; Cole and Hall (2004a) argue for its inclusion as a vesicular cartilage, whereas Witten et al. (2010) consider the notochord as "cartilage-related" (See Hall, 2005, for further discussion). Chondrocytes, or "chondrocyte-like" cells are predominant 
in the mature nucleus pulposus (NP), which is derived from the embryonic notochord. Although historically the embryonically derived notochordal cells are considered to disappear and be replaced from invading cells of another lineage, evidence is growing that demonstrates a single origin of notochordal and NP cells, regardless of their histological appearance (reviewed in Risbud et al., 2010). Kim et al. (2009) demonstrate the ability of NP cells to differentiate into three morphologically distinct phenotypes in culture, including both large vacuolated notochordal cells and the smaller chondrocyte-like cells, whereas Gilson et al. (2010) identify the presence of two seemingly distinct populations of cells within the mature NP, one of which continues to express notochordal markers. Similarly, there is circumstantial evidence that hypertrophic chondrocytes, rather than dying off as is generally presumed, have the capacity, and to at least some extent regularly undergo, proliferation and transdifferentiation into osteoblasts changing their genetic program to express an osteogenic phenotype (reviewed in Wlodarski et al. 2006). Recently Hammond et al. (2009) identify a population of type II collagen expressing hypertrophic chondrocytes in the zebrafish that are differentially responsive to hedgehog signaling, and undergo a switch to an osteogenic phenotype, expressing osteoblast markers including osterix, type I collagen, and osteocalcin. Taken altogether, these data support the notion of a single skeletal cell origin and diversification towards a spectrum of cellular phenotypes. Combined with the fact that similar tissues are present in various invertebrate lineages begs the question: When exactly DID cartilage as a tissue type, or rather the chondrocyte as a cell type, evolve?

It is obvious from analysis of basal metazoan taxa that the capacity to synthesize fibrous protein and mucopolysaccharides in order to obtain a resistant connective tissue is a very ancient feature. However, perhaps the generation of a single cell type that is specialized for the local generation of this type of connective tissue has arisen independently multiple times - or perhaps only once with subsequent diversification (Fig. 1). The question becomes - how can we distinguish between these alternatives? The answer may lie in what has been described by Detlev Arendt as the Molecular Fingerprint of a cell type. The molecular fingerprint is the set of transcription factors, signaling molecules, and structural genes that are specific to a single cell type. Arendt $(2003,2005)$ defines a cell type as a "homogeneous population of cells expressing the same set of orthologous genes for specification and differentiation to implement a defined cellular phenotype". Furthermore, "homologous cell types can be identified by molecular fingerprint comparisons" (Arendt, 2005; Arendt, 2008). Chondrogenesis is most often defined molecularly by the expression of the transcription factor Sox9 and the extracellular matrix molecule collagen type II, however neither of these genes are restricted to cartilage (Cheah et al., 1991; Barrionuevo and Scherer, 2010). Thus, how might one define the molecular fingerprint for the chondrocyte?

\section{Chondrocyte Molecular Fingerprint}

Fig. 2 presents a synthesis of the transcriptional regulation of chondrogenesis in vertebrates, derived from data from the literature regarding regulatory interactions, which has been compiled into a gene regulatory network (GRN) framework. For simplicity, data has been divided into one of four phases corresponding to the developmental progression of chondrogenesis: Mesenchymal expression prior to cellular condensation, cellular condensation, differentiated cartilage, and hypertrophy. This division is arbitrarily over-simplified given the complexity of cartilage maturation, however serves as a necessary starting point to initiate the generation of this complex GRN. It is important to note, that most components of the network are derived from element-specific studies, and thus could reflect region specific patterning sub-modules that interact with the GRN rather than chondrogenesis per se, highlighting the importance of verifying the connectivity between each node. The HOX family of transcription factors has been largely omitted from the current analysis, as these genes are known to act as important patterning determinants and thus are not necessarily applicable to chondrogenesis as a whole. An exception is the Hoxc8 transcription factor, which has been shown to promote the expression of Ncam 1 during pre-cartilage condensation, as well as promoting proliferation, and preventing progression to hypertrophy (Yueh et al., 1998; Lei et al., 2005) although the mechanism of this action remains undefined. A number of studies taking advantage of emerging technologies for looking at global gene expression changes by way of microarray data from microdissected tissues have been published in recent years (i.e., Yamane et al., 2007; Belluccio et al., 2008; Mienaltowski et al., 2008; Zhang et al., 2008; Shapiro et al., 2009; Cameron et al., 2009; Lui et al., 2010). These studies have identified a number of transcription factors for which the role in chondrogenesis is unclear, included here as singlegene nodes to the left of the network. A number of these genes are introduced below.

The transcription factor Sox9 and type II collagen (Col2a1), the most abundant collagen within the cartilage extracellular matrix (ECM), are the two most widely studied components of the chondrogenesis GRN, as evidenced by the large number of inputs on both genes. Both osteocytes and chondrocytes arise from a common mesenchyme "stem" cell which are specified as osteochondro-progenitors through the action of the transcription factor Sox9; Sox9 has been demonstrated to be instrumental in the progression of these cells through the various phases of chondrocyte differentiation. In fact, Sox 9 is the only transcription factor identified to date who's absence leads to the complete loss of all cartilage within the organism - thus highlighting its role as a sort of mastercontrol gene for chondrogenesis. It is important to note that Sox9 is also expressed in many other tissues (testis: Barrionuevo and Scherer (2010); cerebral cortex: Alcock et al. (2009); gastric carcinoma: Zhou et al. (2010); pancreas: Rovira et al. (2010)) and thus although extremely 

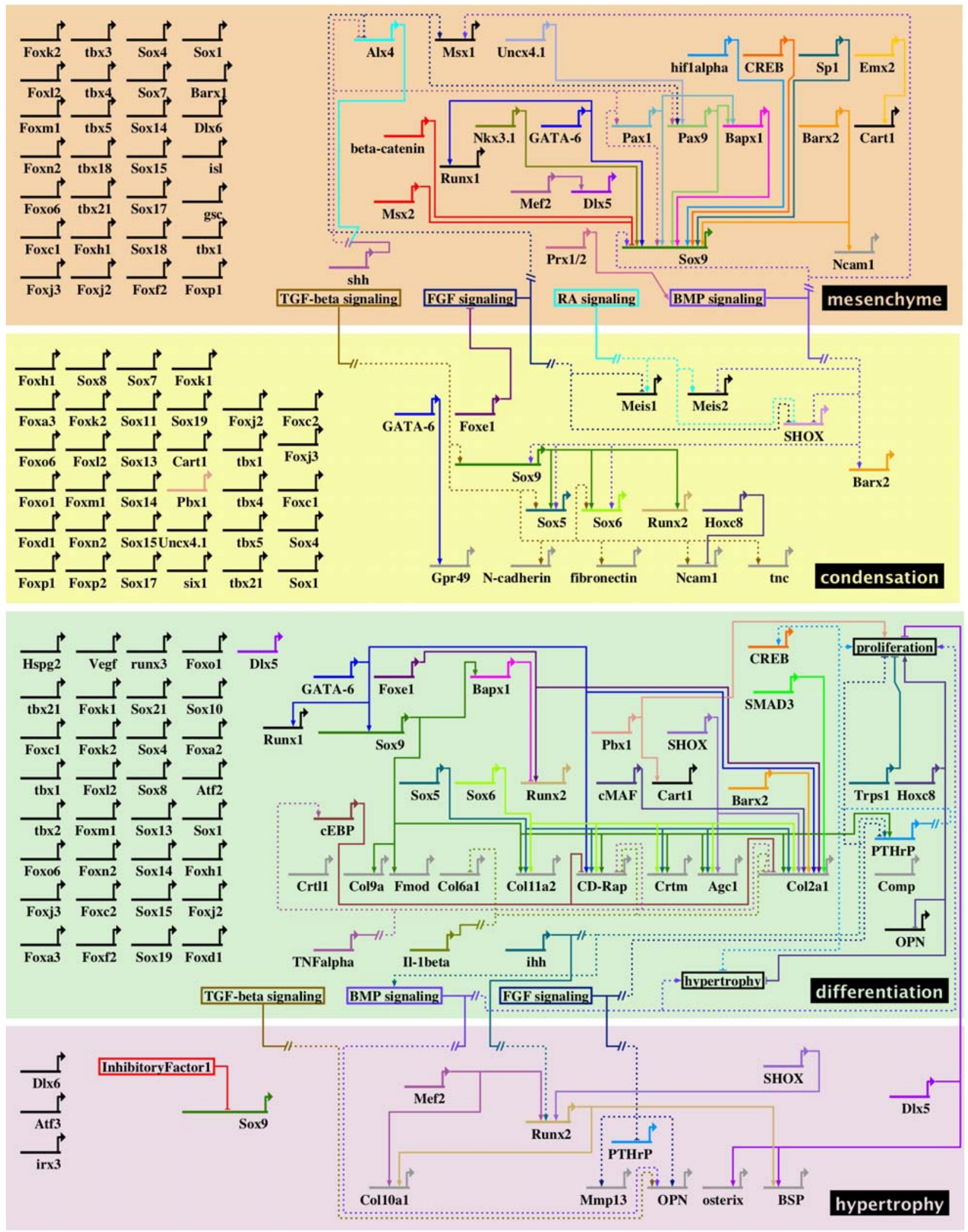

Fig. 2. GRN. Gene regulatory network of vertebrate chondrogenesis. Data regarding genetic interactions involved in the specification and differentiation of vertebrate chondrocytes compiled from published reviews and primary data papers was used as input to the GRN visualization program BioTapestry. Regulatory interactions are separated into four categories: mesenchyme (prior to cellular condensation); condensation; differentiation (onset of extracellular matrix deposition and maturation); hypertrophy (maturation and calcification of extracellular matrix prior to remodeling and bone deposition). Data derived from studies of mature cartilage, wherein expression data involves resting or proliferating chondrocytes, have been included in the differentiated cartilage category. Identification of novel transcription factors by way of comparative microarray studies are included as single-gene nodes to the left of 
the network. Each interaction is shown as a line originating from the source transcription factor of the same color, connecting to the target gene(s). Only transcription factors and known differentiation products are included in the analysis; Signals originating from signaling pathways (BMP, Wnt, FGF, TGF signaling) are included only in the simplest form originating from the mesenchyme and/or during the differentiation phase, and are depicted as passing through a signaling cascade (//). Cell signaling derived interactions are depicted with dotted lines, and occur both within and between developmental phases. Arrowheads represent positive input to the target gene, whereas inhibitory interactions are depicted as $-\mid$ on the target gene. Differentiation factors are located near the bottom of the network and are depicted in grey. Genes active in multiple phases are present in each phase. Interactions depicted are not necessarily direct; in many cases this information is currently unknown.

important for chondrogenesis, is by no means specific for chondrogenesis per se. Nonetheless, Sox 9 is commonly believed to be at the top of the hierarchy of this developmental program, which includes activating the expression of Sox 5, and Sox 6 (Akiyama et al., 2002). Altogether these three transcription factors lead to the upregulation of cartilage matrix components including collagen 2 (Col2a1) and aggrecan (Agc1). A number of transcription factors and signaling pathways are involved in the activation of Sox 9 prior to condensation and overt differentiation of the cartilage matrix, which is known to be blocked by nuclear beta-catenin (eg, Hill et al., 2005). Sox 9 is known to be down-regulated as chondrocytes pass into the hypertrophic phase of development, however the mechanism of this negative regulation is currently unknown (depicted here as "Inhibitory Factor 1").

Cameron et al. (2009) published the first comprehensive study looking at the global gene expression profiles of early chondrogenesis in the mouse hind limb. The authors collected condensing mesenchymal cells, precartilage condensations, and protocartilage cells, and subjected the RNA derived from each of these populations to a genomic microarray analysis to identify changes in gene expression as the mesenchymal cells undergo the transition to chondrocytes. This study revealed 29 transcription factors that were highly expressed in the early condensing mesenchyme, which were subsequently down regulated as the chondrocytes being to express their differentiated phenotype. Conversely, 11 transcription factors were upregulated during this transition (Cameron et al., 2009, additional file 2). Of those expressed before overt differentiation of the chondrocytes, hence presumably active during chondrocyte specification, a number have already been identified as important in chondrocyte specification: alx4 (Qu et al., 1999), barx2 (Jones et al., 1997), msx2 (Takahashi et al., 2001) pax9 (LeClair et al., 1999), and pbx1 (Selleri et al., 2001). Interestingly, Bapx1, Runx2, Runx3, and Dlx5, are upregulated as the chondrocytes enter the differentiation phase (Cameron et al., 2009, additional file 2), suggesting a role for these genes later in the gene regulatory network leading to cartilage formation. Novel transcription factors identified as up-regulated within the condensing mesenchyme include $g s c$, isl1, pitx1, six1, ttf1, sox7, sox17 and eight members of the T-box (tbx) family of transcription factors, five of which show high levels of expression that are significantly down-regulated as the cells progress into the earliest stages of cartilage differentiation.
T-box transcription factors ( $t b x$ ) have not been identified in any other microarray study wherein maturing cartilage has been the tissue examined, further suggesting that $t b x$ genes may serve as negative regulators of chondrogenesis. six1 was also identified as being highly expressed in surface articular cartilage cells with respect to more mature cartilage (Yamane et al., 2007). ttf1 is known to be expressed in tissues immediately adjacent to developing tracheal cartilages (Park et al., 2010); tissues harvested for the microarray study of Cameron et al. (2009) were taken by hand scraping, thus the possibility exists that contamination of non-chondrogenic mesenchyme is present in the analyzed RNA. This illustrates the importance of independent evaluation and validation of microarray data.

As expected from other studies that illustrate the role of Sox HMG transcription factors throughout chondrogenesis (Akiyama et al., 2002; Akiyama, 2008), neither Sox 9 nor Sox 5 and Sox6 were shown to be differentially expressed during the condensation and onset of differentiation phases of cartilage formation, although expression levels of all three genes was elevated, in particular Sox 9 and Sox 6 (Cameron et al., 2009). Other members of the Sox family previously known to be expressed during chondrogenesis include Sox 8, 10 (Chimal-Monroy et al., 2003) and 13 (Wang et al., 2006), part of the Sox E group of transcription factors. All three of these genes were demonstrated to be upregulated as the mesenchymal condensation passes into the initial phase of differentiation. Also identified as being highly expressed in the condensation phase of cartilage specification is the SoxC gene, Sox 11. Sox 11 was also identified as being highly expressed within the growth plate respect to the perichondrium and resting chondrocytes (Yamane et al., 2007). The function of Sox 11 within chondrogenesis has not been identified, although this gene was identified in a microarray screen of osteoarthritic cartilage (Iliopoulos et al., 2008). Interestingly, low levels of an additional seven Sox genes were found throughout chondrogenesis.

Another family of transcription factors identified is the forkhead-box (fox) family. foxe1 is known to interfere with chondrogenesis in the zebrafish by negatively regulating FGF signaling by blocking the expression of the fgfr3 receptor, as well as enhancing Runx 2 expression (Nakada et al., 2009). The function of other fox genes in the context of chondrogenesis is unknown. However Cameron et al. (2009) find a number of these genes to be highly expressed during the condensation phase of chondrogenesis, with 
foxa3, foxo1, and foxd1 showing an increase in expression as the cells enter the differentiation phase. Foxp1 showed slightly elevated expression during cartilage condensation, and was also identified by Yamane et al. (2007) as showing a 6 fold increase in expression within surface articular chondrocytes with respect to the growth plate chondrocytes.

It is evident from the number of previously unreported transcription factors represented in Fig. 2, that much remains to be discovered regarding the gene regulatory network for chondrogenesis in vertebrates, in particular to unravel the minimal regulatory kernels (Davidson and Erwin, 2006; Nowick and Stubbs, 2010) that may represent the ancestral molecular fingerprint for the chondrocyte. The large phenotypic overlap between various vertebrate and invertebrate skeletal cells compounds this problem and the question of the evolutionary origin of these cell types remains (Fig. 1). There are at least three possibilities: that chondrocytes are homologous across lineages and thus cartilage is a homologous tissue. That some chondrocytes in some lineages are homologous. Or that all chondrocytes in different lineages are the result of independent, convergent evolution. To distinguish between these alternatives, detailed molecular analyses of non-vertebrate cartilages is required.

\section{Molecular Studies of Invertebrate Cartilages}

These types of analyses have been carried out independently by various groups, leading to somewhat different conclusions. Wada (2010) recently pulled together an analysis of deuterostome genomic information to conclude that at least within the deuterostomes there is strong evidence for an ancestral gene regulatory network where Sox genes regulate the expression of fibrous collagens. Both SoxE and Runx homologs are present within the cellular cartilaginous skeletons of non-vertebrate chordates (lamprey and hagfish: McCauley and BronnerFraser, 2006; Zhang et al., 2006; Ohtani et al., 2008; Hecht et al., 2008). Presently the only non-chordate invertebrates to be investigated have only an acellular branchial skeleton. Although SoxE was found to be expressed within the hemichordate skeleton (Rychel and Swalla, 2006), it is absent within the skeletal tissues of the cephalochordate, while present within the notochord (Meulemans and Bronner-Frazer, 2007) as is the Runx gene (Hecht et al., 2008). The absence of the chondrocyte in these systems precludes robust conclusions regarding the ancestral nature of a chondrocyte gene regulatory network; given the ease with which things can be lost over time versus the challenges in re-inventing the wheel, so to speak, we might conclude that absence of evidence is by no means evidence of absence.

The invertebrate system that may be the most adapt for addressing the question of the evolutionary origins of the chondrocyte as a cell type is the European cuttlefish, Sepia officinalis. This animal has 13 different skeletal elements that have been described as cartilaginous. Of these, histological analysis reveals that more than half of them correspond histologically to hyaline cartilage (Cole and Hall, 2009). The development of these cartilaginous elements is somewhat variable despite the lack of differences in the histology of the mature tissue: Most of these cartilages develop as a metaplasia from uncondensed mesenchymal tissue, however a number of the more prominent, and phylogenetically conserved amongst cephalopod lineages, go through a condensation phase similar to that described for vertebrates. And of these, a number of elements develop from a condensation that directly underlies a cuboidal epithelial layer. The sepia hedgehog ortholog is expressed in association with some cartilaginous tissues (Navet et al., 2009) in particular within the overlying cuboidal epithelial layer prior to the overt differentiation of the underlying cartilage (L. Focareta and A.G. Cole, unpublished data), suggesting that this important signaling molecule may be involved in the early specification and/or patterning of cuttlefish cartilage formation. A detailed molecular analysis of this cuttlefish cartilage is currently underway with the goal of addressing the level of homology, if any, that exists between cellular cartilage from an invertebrate model and vertebrate cartilage.

\section{Perspectives}

Cartilage is a complex tissue type, whose complexity is enhanced by the existence of numerous developmental phases and overlap in phenotypic gene expression with related cell types (i.e. notochordal cells and osteoblasts). The GRN for vertebrate chondrogenesis presented here serves as an initial synthesis of the molecular data available and highlights a number of potentially important transcription factors that deserve further attention from the scientific community. It is hoped that this presentation format will serve to assist in the design of further microarray studies aimed at clarifying, and verifying, the genetic interactions underlying cartilage formation, as well as serving as a platform for comparative studies in nonvertebrate lineages with the goal of uncovering the evolutionary origin of this complex and important tissue type.

Furthermore, invertebrate cartilages, particularly those found within cephalopods, may represent novel animal models for the study of cartilage from the prospective of evolution at the level of gene regulatory networks and molecular fingerprints. The presence of cartilaginous tissues in invertebrates opens new avenues for the exploration of biomechanical properties of cartilage and have the capacity to enlighten us on mechanisms of cartilage regeneration, two areas that have so far gone unexplored.

\section{Acknowledgements}

Ongoing molecular characterization of Sepia officinalis cartilage is supported by a European Commission grant: Mari Curie FP7-PEOPLE-2009-RG; 246680. 


\section{References}

Abbott J, Holtzer H (1966) The loss of phenotypic traits by differentiated cells. 3 . The reversible behavior of chondrocytes in primary cultures. J Cell Biol 28: 473-487.

Akiyama H (2008) Control of chondrogenesis by the transcription factor Sox9. Mod Rheumatol 18: 213-219.

Akiyama H, Chaboissier M, Martin JF, Schedl A, de Crombrugghe B (2002) The transcription factor Sox9 has essential roles in successive steps of the chondrocyte differentiation pathway and is required for expression of Sox5 and Sox6. Genes Dev 16: 2813-2828.

Alcock J, Lowe J, England T, Bath P, Sottile V. (2009) Expression of Sox1, Sox2 and Sox9 is maintained in adult human cerebellar cortex. Neurosci Lett 450: 114-116.

Arendt D (2003) Evolution of eyes and photoreceptor cell types. Int J Dev Biol 47: 563-571.

Arendt D (2005) Genes and homology in nervous system evolution: comparing gene functions, expression patterns, and cell type molecular fingerprints. Theory Biosci 124: 185-197.

Arendt D (2008) The evolution of cell types in animals: emerging principles from molecular studies. Nat Rev Genet 9: $868-882$.

Bairati A, Comazzi M, Cioria M, Rigo C (1998) The ultrastructure of chondrocytes in the cartilage of Sepia officinalis and Octopus vulgaris (Mollusca, Cephalopoda). Tissue Cell 30: 340-351.

Barrionuevo F, Scherer G (2010) SOX E genes: SOX9 and SOX8 in mammalian testis development. Int J Biochem Cell Biol 42: 433-436.

Belluoccio D, Bernardo BC, Rowley L, Bateman JF (2008) A microarray approach for comparative expression profiling of the discrete maturation zones of mouse growth plate cartilage. Biochim Biophys Acta 1779: 330-340.

Benjamin M (1990) The cranial cartilages of teleosts and their classification. J Anat 169: 153-172.

Cameron TL, Belluoccio D, Farlie PG, Brachvogel B, Bateman JF (2009) Global comparative transcriptome analysis of cartilage formation in vivo. BMC Dev Biol 9: 20.

Chacko S, Abbott J, Holtzer S, Holtzer H. (1969) The loss of phenotypic traits by differentiated cells. VI. Behavior of the progeny of a single chondrocyte. J Exp Med 130: 417-442.

Cheah K, Lau ET, AU PKC, Tam PPL (1991) Expression of the mouse a1(11) collagen gene is not restricted to cartilage during development. Development 111: 945-953.

Chimal-Monroy J, Rodriguez-Leon J, Montero JA, Ganan Y, Macias D, Merino R, Hurle JM. (2003) Analysis of the molecular cascade responsible for mesodermal limb chondrogenesis: Sox genes and BMP signaling. Dev Biol 257: 292-301.

Cole AG, Hall BK (2004a) Cartilage is a metazoan tissue; integrating data from non-vertebrate sources. Acta Zoologica 85: 69-80.

Cole AG, Hall BK (2004b) The nature and significance of invertebrate cartilages revisited: distribution and histology of cartilage and cartilage-like tissues within the Metazoa. Zoology 107: 261-273
Cole AG, Hall BK (2009) Cartilage differentiation in cephalopod molluscs. Zoology 112: 2-15

Davidson EH, Erwin DH (2006) Gene regulatory networks and the evolution of animal body plans. Science 311: 796-800.

Gilson A, Dreger M, Urban JP (2010) Differential expression level of cytokeratin 8 in cells of the bovine nucleus pulposus complicates the search for specific intervertebral disc cell markers. Arthritis Res Ther 12: R24.

Hall BK (2005) Bones and cartilage: developmental and evolutionary skeletal biology. Elsevier D†Academic Press, London, 760 pp.

Hall BK, Miyake T (2000) All for one and one for all: condensations and the initiation of skeletal development. BioEssays 22: 138-147.

Hammond CL, Schulte-Merker S (2009) Two populations of endochondral osteoblasts with differential sensitivity to Hedgehog signalling. Development 136: 3991-4000

Hecht J, Stricker S, Wiecha U, Stiege A, Panopoulou G, Podsiadlowski L, Poustka AJ, Dieterich C, Ehrich S, Suvorova J, Mundlos S, Seitz V. (2008) Evolution of a core gene network for skeletogenesis in chordates. PLoS Genet 4: e1000025.

Hill TP, Später D, Taketo MM, Birchmeier W, Hartmann C (2005) Canonical Wnt/beta-catenin signaling prevents osteoblasts from differentiating into chondrocytes. Dev Cell 8: 727-738.

Iliopoulos D, Malizos KN, Oikonomou P, Tsezou A (2008) Integrative microRNA and proteomic approaches identify novel osteoarthritis genes and their collaborative metabolic and inflammatory networks. PLoS ONE 3: e3740.

Jones FS, Kioussi C, Copertino DW, Kallunki P, Holst BD, Edelman GM (1997) Barx2, a new homeobox gene of the Bar class, is expressed in neural and craniofacial structures during development. Proc Natl Acad Sci USA 94: 2632-2637.

Kim JH, Deasy BM, Seo HY, Studer RK, Vo NV, Georgescu HI, Sowa GA, Kang JD (2009) Differentiation of intervertebral notochordal cells through live automated cell imaging system in vitro. Spine 34: 2486-2493.

Kupriyanova EK, Rouse GW (2008) Yet another example of paraphyly in Annelida: molecular evidence that Sabellidae contains Serpulidae. Mol Phylogenet Evol 46: 1174-1181.

LeClair EE, Bonfiglio L, Tuan RS (1999) Expression of the paired-box genes Pax-1 and Pax-9 in limb skeleton development. Dev Dyn 214: 101-115.

Lei H, Wang H, Juan AH, Ruddle FH (2005) The identification of Hoxc8 target genes. Proc Natl Acad Sci USA 102: 2420-2424

Leone F, Bairati A, Vitellaro-Zuccarello L (2004) The cytoskeleton of chondrocytes of Sepia officinalis (Mollusca, Cephalopoda): an immunocytochemical study. Eur J Histochem 48: 159-166.

Lui JC, Andrade AC, Forcinito P, Hegde A, Chen W, Baron J, Nilsson O (2010) Spatial and temporal regulation of gene expression in the mammalian growth plate. Bone 46: 1380-1390. 
McCauley DW, Bronner-Fraser M (2006) Importance of SoxE in neural crest development and the evolution of the pharynx. Nature 441: 750-752.

Meulemans, Bronner-Fraser M (2007) Insights from amphioxus into the evolution of vertebrate cartilage. PLosOne 8: e787.

Mienaltowski MJ, Huang L, Stromberg AJ, MacLeod JN (2008) Differential gene expression associated with postnatal equine articular cartilage maturation. BMC Musculoskelet Disord 9: 149.

Murray DH, Bush PG, Brenkel IJ, Hall AC (2010) Abnormal human chondrocyte morphology is related to increased levels of cell-associated IL- $1 \beta$ and disruption to pericellular collagen type VI. J Orthoped Res, in press.

Nakada C, Iida A, Tabata Y, Watanabe S (2009) Forkhead transcription factor foxe1 regulates chondrogenesis in zebrafish. J Exp Zool (Mol Dev Evol) 312B: 827-840.

Navet S, Andouche A, Baratte S, Bonnaud L (2009) Shh and Pax6 have unconventional expression patterns in embryonic morphogenesis in Sepia officinalis (Cephalopoda). Gene Expr Patterns 9: 461-467.

Nowick K, Stubbs L (2010) Lineage-specific transcription factors and the evolution of gene regulatory networks. Brief Funct Genomics 9: 65-78.

Ohtani K, Yao T, Kobayashi M, Kusakabe R, Kuratani S, Wada H (2008) Expression of Sox and fibrillar collagen genes in lamprey larval chondrogenesis with implications for the evolution of vertebrate cartilage. J Exp Zool (Mol Dev Evol) 310B: 596-607.

Park J, Zhang JJ, Moro A, Kushida M, Wegner M, Kim PC (2010) Regulation of Sox 9 by Sonic Hedgehog (Shh) is essential for patterning and formation of tracheal cartilage. Dev Dyn 239: 514-526.

Person, P, Philpott, DE (1969) The nature and significance of invertebrate cartilages. Biol Rev Camb Philos Soc 44: 1-16.

Qu S, Tucker SC, Zhao Q, deCrombrugghe B, Wisdom $\mathrm{R}(1999)$ Physical and genetic interactions between Alx4 and Cart1. Development 126: 359-369.

Risbud MV, Schaer TP, Shapiro IM (2010) Toward an understanding of the role of notochordal cells in the adult intervertebral disc: from discord to accord. Dev Dyn 239: 2141-2148.

Rovira M, Scott SG, Liss AS, Jensen J, Thayer SP, Leach SD (2010). Isolation and characterization of centroacinar/terminal ductal progenitor cells in adult mouse pancreas. Proc Natl Acad Sci USA 107: 75-80.

Rychel AL, Swalla BJ (2007) Development and evolution of chordate cartilage. J Exp Zool (Mol Dev Evol) 308B: 325-335.
Selleri L, Depew MJ, Jacobs Y, Chanda SK, Tsang KY, Cheah KSE, Rubenstein JLR, O'Gorman S, Cleary ML (2001) Requirement for Pbx1 in skeletal patterning and programming chondrocyte proliferation and differentiation. Development 128: 3543-3557.

Shapiro F, Flynn E, Calicchio ML (2009) Molecular differentiation in epiphyseal and physeal cartilage. Prominent role for gremlin in maintaining hypertrophic chondrocytes in epiphyseal cartilage. Biochem Biophys Res Commun 390: 570-576.

Takahashi K, Nuckolls GH, Takahashi I, Nonaka K, Nagata M, Ikura T, Slavkin HC, Shum L (2001) Msx2 is a repressor of chondrogenic differentiation in migratory cranial neural crest cells. Dev Dyn 222: 252-262.

Wada H (2010) Origin and genetic evolution of the vertebrate skeleton. Zoolog Sci 27: 119-123.

Wang Y, Ristevski S, Harley VR (2006) SOX13 exhibits a distinct spatial and temporal expression pattern during chondrogenesis, neurogenesis, and limb development. J Histochem Cytochem 54: 1327-1333.

Witten PE, Huysseune A, Hall BK (2010) A practical approach for the identification of the many cartilaginous tissues in teleost fish. J Appl Ichthyol 26: 257-262.

Wlodarski KH, Wlodarski PK, Brodzikowska A. (2006) Metaplasia of chondrocytes into osteoblasts. Folia Biol (Krakow) 54: 75-80.

Yamane S, Cheng E, You Z, Reddi AH (2007) Gene expression profiling of mouse articular and growth plate cartilage. Tissue Eng 13: 2163-2173.

Yueh YG, Gardner DP, Kappen C (1998) Evidence for regulation of cartilage differentiation by the homeobox gene Hoxc-8. Proc Natl Acad Sci USA 95: 9956-9961.

Zhang G, Miyamoto MM, Cohn MJ (2006) Lamprey type II collagen and Sox9 reveal an ancient origin of the vertebrate collagenous skeleton. Proc Natl Acad Sci USA. 103: $3180-3185$.

Zhang M, Pritchard MR, Middleton FA, Horton JA, Damron TA (2008) Microarray analysis of perichondral and reserve growth plate zones identifies differential gene expressions and signal pathways. Bone 43: 511-520.

Zhou CJ, Guo JQ, Zhu KX, Zhang QH, Pan CR, Xu WH, Wang HJ, Liu B (2010) Elevated expression of SOX9 is related with the progression of gastric carcinoma. Diagn Cytopathol, in press.

Editor's Note: Since there were no questions from the reviewers, there is no "Discussion with Reviewers" section. 